\title{
Shared decision making
} and patient-centeredness for patients with poorly controlled type 2 diabetes mellitus in primary care-results of the cluster-randomised controlled DEBATE trial

Anja Wollny' ${ }^{1}$ Christin Löffler ${ }^{1 *}$, Eva Drewelow ${ }^{1}$, Attila Altiner ${ }^{1}$, Christian Helbig ${ }^{1}$, Anne Daubmann², Karl Wegscheider ${ }^{2}$, Susanne Löscher ${ }^{3}$, Michael Pentzek ${ }^{3}$, Stefan Wilm ${ }^{3}$, Gregor Feldmeier ${ }^{1}$ and Sara Santos ${ }^{3}$

\begin{abstract}
Background: We investigate whether an educational intervention of GPs increases patient-centeredness and perceived shared decision making in the treatment of patients with poorly controlled type 2 diabetes mellitus?

Methods: We performed a cluster-randomized controlled trial in German primary care. Patients with type 2 diabetes mellitus defined as $\mathrm{HbA} 1 \mathrm{c}$ levels $\geq 8.0 \%(64 \mathrm{mmol} / \mathrm{mol})$ at the time of recruitment $(n=833)$ from general practitioners $(n=108)$ were included. Outcome measures included subjective shared decision making (SDM-Q-9; scale from 0 to 45 (high)) and patient-centeredness (PACIC-D; scale from 1 to 5 (high)) as secondary outcomes. Data collection was performed before intervention (baseline, T0), at 6 months (T1), at 12 months (T2), at 18 months (T3), and at 24 months (T4) after baseline.

Results: Subjective shared decision making decreased in both groups during the course of the study (intervention group: -3.17 between T0 and T4 (95\% Cl: $-4.66,-1.69 ; p<0.0001)$ control group: -2.80 (95\% Cl: $-4.30,-1.30 ; p=0.0003)$ ). There were no significant differences between the two groups $(-0.37 ; 95 \% \mathrm{Cl}:-2.20,1.45 ; p=0.6847)$. The intervention's impact on patient-centeredness was minor. Values increased in both groups, but the increase was not statistically significant, nor was the difference between the groups.

Conclusions: The intervention did not increase patient perceived subjective shared decision making and patientcenteredness in the intervention group as compared to the control group. Effects in both groups might be partially attributed to the Hawthorne-effect. Future trials should focus on patient-based intervention elements to investigate effects on shared decision making and patient-centeredness.
\end{abstract} original author(s) and the source, provide a link to the Creative Commons licence, and indicate if changes were made. The images or other third party material in this article are included in the article's Creative Commons licence, unless indicated otherwise in a credit line to the material. If material is not included in the article's Creative Commons licence and your intended use is not permitted by statutory regulation or exceeds the permitted use, you will need to obtain permission directly from the copyright holder. To view a copy of this licence, visit http://creativecommons.org/licenses/by/4.0/. The Creative Commons Public Domain Dedication waiver (http://creativeco mmons.org/publicdomain/zero/1.0/) applies to the data made available in this article, unless otherwise stated in a credit line to the data. 
Trial registration: The trial was registered on March 10 $0^{\text {th }}, 2011$ at ISRCTN registry under the reference ISRCTN7071 3571.

Keywords: Diabetes mellitus type 2, Physician-patient relations, Decision making, Health communication, Health services research

\section{Background}

The rising prevalence of age-associated diseases is a major consequence of increasing life expectancy $[1,2]$. Globally, diabetes mellitus is among the most important metabolic disorders and has become a major health care burden [3, 4]. Since 1980, lifetime prevalence of diabetes mellitus showed a major increase with about $6.3 \%$ of adults being affected in $2017[3,5,6]$.

Among patients with type 2 diabetes mellitus (T2DM) registered in the German Disease Management Programme (DMP), $10 \%$ show poor control [7]. Studies indicate that various aspects influence the success of treatment and can often compromise adherence. Most common factors include psychosocial stressors, the nature of the disease with e.g., the absence of symptoms, the presence of comorbidities, as well as complex therapy plans poorly adjusted to the patient's individual situation [8-12]. For the majority of patients, diabetes mellitus is managed by general practitioners (GPs) [13]. From their perspective, treating patients with poorly controlled T2DM is rarely successful and often leads to frustration. This is mainly related to differences in the preferred disease management between patients and doctors. A lack of patient-centred communication reinforces this tendency [14-20].

However, patients increasingly wish to be more involved in decision making [21]. Shared decision making was also shown to be beneficial for physicians` satisfaction with consultations: It allows GPs to gain more insight into the concerns, emotions and needs of their patients [22]. Elicitation and appreciation of patients' views on their disease allows doctors to better understand their patients' behaviour and enables them to align treatment plans with their patients' disease concepts in mind [23-25].

The aim of this study was to investigate the effects of an intervention that facilitates patient-centeredness and shared decision making among GPs and their patients with poorly controlled type 2 diabetes mellitus in German primary care. This paper illustrates the secondary outcomes subjective shared decision making and patient-centeredness.

\section{Methods}

\section{Trial design}

The DEBATE trial is a cluster-randomised controlled trial testing the effect of an educational intervention on the management of patients with poorly controlled T2DM. Primary outcome was the level of HbA1c. Secondary outcomes were patient-centeredness and subjective shared decision making. Data was collected before randomization (baseline, T0), at 6 months (T1), at 12 months (T2), at 18 months (T3), and at 24 months (T4) after baseline. At each point we allowed a maximum of two additional months for data collection e.g., in case where patients could not be reached.

The primary outcome measure (level of HbA1c) decreased significantly both in the intervention and control group. The effect, however, was not significantly different between both groups. Results are published elsewhere [26].

\section{Recruitment, eligiblity criteria and sampling procedure}

For recruitment registers of the regional Associations of Statutory Health Insurance Physicians of the German regions Mecklenburg-Western Pomerania and North Rhine-Westphalia were used. Among participating practices of general practice patients affected by T2DM with an HbA1c level of $\geq 8.0 \%(64 \mathrm{mmol} / \mathrm{mol})$ within three months prior to recruitment were contacted and included. Patients with one of the following characteristics were excluded from participation: living with a severe comorbidity resulting in an assumed life expectancy below 24 months, inability to give informed consent or lack of sufficient German language skills.

Detailed information on the process of recruitment is published elsewhere [27].

\section{Intervention}

Based on qualitative data from German primary care we know that GPs perceive their patients with poorly controlled T2DM as in denial or refusing to follow advice. In this situation, GPs are sometimes inclined to lower expectations for improvement. In some cases, they also became resigned to the situation [16]. To address this pattern we developed an interventional 
concept that encourages patient-centered communication and shared decision making.

In a first step, GPs specially trained in patient-centered communication visited enrolled GPs. This peer-visit aimed at sensitizing for patients' concepts of disease and their views, attitudes, and behaviors by using patientcentered communication. In a further step, GPs were encouraged to use the electronic decision-aid (https:// www.arriba-hausarzt.de/) to increase shared decision making. The decision-aid uses HbA1c levels and associated risk factors to visualize the probability of experiencing macro vascular events $[28,29]$. The effect of antidiabetic medication (such as oral or insulin therapy) and lifestyle changes on cardiovascular events are also shown and serve as a starting point for shared decision making.

A total of 54 GPs were randomized into the intervention group. Out of these after baseline data collection 47 GPs were visited by peers (component 1 ). Seven GPs were not available for the visit. They received written information material and a phone call. In addition to that, enrolled GPs were provided the chance to take part in a workshop on patient-centered communication. A total of 10 GPs took up the offer (component 2). Please find a detailed description of the intervention components in Tables 1 and 2.

\section{Control}

Patients of the control group received care as usual. In Germany, this usually includes measuring levels of
HbA1c and consulting the GP two to four times a year. GPs of the control group did not receive any form of intervention.

\section{Outcomes}

Primary outcome was the change of HbA1c level from T0 to T4, which was statistically significant within each group, but not between groups. Results have been published elsewhere [26].

Secondary endpoints included subjective shared decision making (SDM-Q-9 [30]) and patient-centeredness (PACIC-D [31]). Members of the study team collected data by phone at the different points of measurements. Detailed information on data collection procedures was published elsewhere [26, 27].

Analysis of perceived extent of subjective shared decision making from the perspective of T2DM patients was conducted through SDM-Q-9. The questionnaire is based on a 6 -stage Likert-scale $(0=$ does not apply at all; $5=$ fully applies). An overall score resulted from the addition of all nine items, giving a scale from 0 to 45 . High values indicate that the extent of shared decision making was perceived as high by patients [30].

Patient-centeredness was measured through the short version of the PACIC-D questionnaire that contains 11 items. However, given the age of patients and ease of use, we decided for one modification. Instead of using the scale of the short version (percentage between 0 and 100 , in steps of 10 percent), we adapted the five-stage Likert-scale of the long version PACIC-D questionnaire

Table 1 Intervention description of component 1: outreach educational peer visit (according to TIDieR)

\begin{tabular}{|c|c|}
\hline 1 Short Name & Educational peer visit \\
\hline 2 Goal and rationale & $\begin{array}{l}\text { Improvement of doctor-patient communication and interaction between GP and patient, raising GP awareness for patients } \\
\text { with poorly controlled diabetes type 2, their individual agenda and concepts of disease and taking it into account in the } \\
\text { process of shared decision making, putting more focus on the patient perspective without overstraining both, doctor } \\
\text { and patient }\end{array}$ \\
\hline 3 Materials & Oral input, computer-based decision-aid tool arriba-debate, peer-to-peer-discussion \\
\hline 4 Procedures & $\begin{array}{l}\text { Trained GPs visited participating GPs in their practice. During the visitation, specific problems/factors influencing the } \\
\text { doctor-patient-communication and the treatment of patients with poorly controlled type } 2 \text { diabetes were discussed with } \\
\text { the GP (e.g. different ideas of therapy on GPs and patient's sides resulting in ineffective doctor-patient communication, } \\
\text { lack of interest, resignation, frustration, anger). In addition, the peer GP introduced the basics of narrative based com- } \\
\text { munication to the GP and gave individual feedback to patient cases the GP had experienced to be difficult. Addition- } \\
\text { ally, during the visitation, the computer-based decision-aid tool arriba-debate was introduced to the GP. The tool offers } \\
\text { patient-targeted visualizations of the effect of possible behaviour changes (e.g. smoking stop, exercise) and therapy } \\
\text { (medication) on the individual risk of coronary heart disease under consideration of individual parameters (e.g. sex, age, } \\
\text { blood pressure, cholesterol, blood glucose level) }\end{array}$ \\
\hline 5 Providers of intervention & Trained general practitioners (peers) \\
\hline 6 Mode of delivery & On site visit, oral presentation, introduction of the decision-aid tool and discussion \\
\hline 7 Location & GP practice \\
\hline 8 Frequency & $\begin{array}{l}\text { Once following completion of baseline data collection between the } 3 \text { rd quarter of } 2012 \text { and the } 1 \text { st quarter of } 2013 \text {; dura- } \\
\text { tion approximately } 1-1.5 \mathrm{~h} \text {, total of } 47 \text { intervention practices received a peer visit }\end{array}$ \\
\hline 9 Planned tailoring & No \\
\hline 10 Fidelity enhancement & Memo written by peer \\
\hline
\end{tabular}


Table 2 Intervention description of component 2: additional training on patient communication skills for GPs—optional (according to TIDieR)

\begin{tabular}{|c|c|}
\hline 1 Short Name & Additional training for GPs to promote patient-centred communication \\
\hline 2 Goal and rationale & $\begin{array}{l}\text { Exploration of individual patient expectations, concepts of disease and barriers in the process of shared decision making in } \\
\text { patients with poorly controlled diabetes type } 2\end{array}$ \\
\hline 3 Materials & $\begin{array}{l}\text { Theoretical input on narrative-based communication, group training on practical use of these skills, computer-based } \\
\text { decision-aid arriba-debate }\end{array}$ \\
\hline 4 Procedures & $\begin{array}{l}\text { Introduction of theoretical background on narrative-based communication (incl. three-step-conversation). Group training } \\
\text { sessions (max. 3-4 participants) under considerations of personal experiences and defaulted roles. The issues of the } \\
\text { sequences differed, starting with a low-threshold one (e.g. vacation), followed by the experience of an in-acute disease } \\
\text { (e.g. cold), ending with a practical oriented issue (e.g. GP as protagonist in the practice). Roles were changed after every } \\
\text { session (narrator, asker, observer) to give all participants the opportunity to slip in each role. Subsequently, feedback } \\
\text { about the practical implementation was given and discussion about transferability in daily routine was carried out } \\
\text { Finally, the computer-based decision-aid tool arriba-debate and its use in daily routine in the GP-practice was discussed }\end{array}$ \\
\hline 5 Providers of intervention & The training was performed by qualified scientific researchers of the study sites in Rostock, Düsseldorf and Witten \\
\hline 6 Mode of delivery & $\begin{array}{l}\text { Single intervention } \\
10 \text { out of the } 54 \text { GPs in the intervention group of DEBATE }\end{array}$ \\
\hline 7 Location & $\begin{array}{l}\text { The training was performed in two of the study sites } \\
\text { Total of five trainings with altogether } 10 \mathrm{GPs} \text { were performed }\end{array}$ \\
\hline 8 Frequency & Each training lasted about $3 \mathrm{~h}$ \\
\hline 9 Planned tailoring & No \\
\hline 10 Fidelity enhancement & \\
\hline
\end{tabular}

( $1=$ almost never, $5=$ nearly always). The overall score was calculated as the mean. A high extent of patientcenteredness in the GP setting from the patient's point of view was reflected in a high value [31-34].

\section{Sample size}

Sample size was determined to ensure statistical significance of primary outcomes and was published elsewhere $[26,27]$.

\section{Stopping rules}

There were no stopping rules within the DEBATE trial.

\section{Randomization}

Randomization was implemented by the study centres after the baseline data collection. Within each study centre, unrestricted randomization was used to allocate the intervention to GPs (cluster level) and their selected patients. Randomization was performed by statisticians not involved in the trial management.

\section{Allocation concealment mechanism}

At recruitment and baseline data collection enrolled GPs were not aware of group allocation.

\section{Blinding}

Study participants were not explicitly blinded. Nonetheless, neither study nurses performing data collection (questionnaires), nor patients were aware of group allocation.

\section{Measures against bias}

To reduce bias related to multiple testing we undertook several preventive measures. These included a standardized interviewer training for all interviewers. Whenever possible, study nurses interviewed the same participants over all points of measurement. Also, questionnaires were not provided before phone interviews and instruments varied to some extent between different points of measurement. Finally, the time interval between measurements was large (six months) and questions always referred to the current day or to the previous six months (see protocol [27]).

\section{Statistical methods}

The chosen versions of SDM-Q-9 and PACIC-D are commonly used in Germany. Initially, descriptive statistics, e.g., mean and standard deviations or absolute and relative frequencies were determined group-wise for each time of measurement.

A longitudinal mixed model with patients and practice as random effects was calculated to account for potential effects of intervention and covariates on changes in SDM-Q-9 and PACIC-D. Follow-ups were incorporated into the model as repeated measurements without restrictions of the covariance matrix. Baseline measurements were included in the model to reduce variance. For control of potential biases, the variables 'study centre' and 'time of measurement' were included as fixed effects. Marital status, age at initial diagnosis, number of persons in the household and cardiovascular 
risk were considered as potential covariates on patient level as well as averaged on practice level, complemented by physician's age. To select these variables, dependent on the scale level of the respective baseline variable, a model with group and study centre as fixed effects was calculated. All variables with a p-value less than 0.2 were included in the next step. In the final set of covariates, that was selected by likelihood ratio based forward selection, only age at diagnosis on patient level was selected as an additional covariate. The time by group interaction was to be tested initially to include this in the model if the interaction was significant. The coefficient test, comparing the adjusted values between the randomized groups, was performed using the direct maximum likelihood as the statistical estimation procedure, which results in unbiased estimators under the missing-at-random-assumption. The analysis was repeated in the per protocol (PP) population. To assess the association between patient-centeredness and subjective shared decision making, we adapted the above described model. SDM was the outcome and changes to baseline in PACIC-D were an additional covariate. The other aspects of the model remained unchanged. Adjusted means with 95\% confidence intervals and $\mathrm{p}$ values were reported. The significance level was set at two-sided $\alpha$-level of 0.05 . All analyses were conducted with SAS, version 9.4 (SAS Institute Inc. Cary, North Carolina, USA).

\section{Results}

\section{Recruitment and participant flow}

Over the period of 24 months (from 08/2011 to 07/2013), 833 patients of 108 GPs were included in the study. 54 GPs (435 patients) were randomized to the intervention group, and 54 GPs (398 patients) were randomized to the control group. At T4 (24 months), a total of 644 patients remained in the study (339 patients intervention group, 305 control group). Patient drop-outs were 22.1\% (intervention) and 23.4\% respectively (control). Most drop-outs were related to a change of GP (21.7\%), death $(21.2 \%)$ or a not primarily diabetes related deterioration of the patients' health status (28\%). Other reasons included patients' loss of interest in the trial and failed attempts to recontact trial participants. See Fig. 1 for the detailed flow of participants.

\section{Baseline data}

Basic socio-demographic and health data of participating patients are provided in Table 3 . There were no considerable differences between groups.

\section{Outcomes}

\section{Subjective shared decision making (SDM-Q-9)}

The intention-to-treat analysis showed that in the intervention group the mean value of the SDM-Q-9 was 23.68 at baseline. Over the course of the study, the value decreased and then slightly increased while remaining below baseline (20.91 at T4). A similar trend was evident in the adjusted model: the difference to baseline was largest at T1 $(-3.57$ (95\% CI: $-4.98 ;-2.17))$ and decreased towards T4 to -3.17 (95\% CI: $-4.66 ;-1.69)$. Within the intervention group, at all times the change in mean SDM-Q-9 values was statistically significant $(p<0.0001)$. Results in the control group were similar: the SDM-Q-9 mean value decreased from 22.42 at baseline to 19.77 at T4. The adjusted difference was maximal at T1 $(-3.20$ (95\% CI: $-4.62 ;-1.78)$ ) and decreased to -2.80 at T4 $(95 \%$ CI: $-4.30 ;-1.30)$. Likewise, changes from baseline were statistically significant at all times $(p<0.0003)$. However, between the groups no significant difference was found $(p=0.6847)$. See Table 4 and Fig. 2.

Table 5 shows the results of the per-protocol analysis. Apart from small deviations, the results followed the same trends as in the intention-to-treat analysis.

\section{Patient-centeredness (PACIC-D)}

As to patient-centeredness, in the intervention group the intention-to-treat analysis showed an average nonadjusted PACIC-D score of 2.42. Over the course of the study, the score slightly increased to 2.52 at T4. The adjusted difference to baseline varied from 0.03 at $\mathrm{T} 1$ (95\% CI: $-0.05 ; 0.12$ ) to 0.09 at T3 (95\% CI: $0.00 ; 0.18$ ), and finally to 0.04 at T4 $(95 \% \mathrm{CI}:-0.05 ; 0.13)$. None of the differences were statistically significant. The same was observed in the control group. The non-adjusted PACIC-D score at T0 was 2.39 and reached the highest value of 2.52 at T3. The adjusted difference to baseline varied from 0.02 at $\mathrm{T} 1$ (95\% CI: $-0.06 ; 0.11)$ to 0.08 at T3 (95\% CI: -0.01; 0.17). None of the corresponding p-values indicated statistical significance. Also, there was no significant difference between the intervention and control group ( $p=0.8677)$. See Table 6 and Fig. 3 .

The per-protocol analysis supported the trend observed in the intention-to-treat analysis. There was however one exception: at T3, the adjusted difference of the PACICD score to baseline was statistically significant in the intervention group: the PACIC-D score increased by 0.12 (95\% CI: 0.02; 0.22, $p=0.02$ ). See Table 7.

As far as the five dimensions of PACIC-D are concerned, we performed descriptive analyses and found differences between intervention and control group for the dimension "coordination and follow-up". Within the intervention group the dimension-specific mean value 


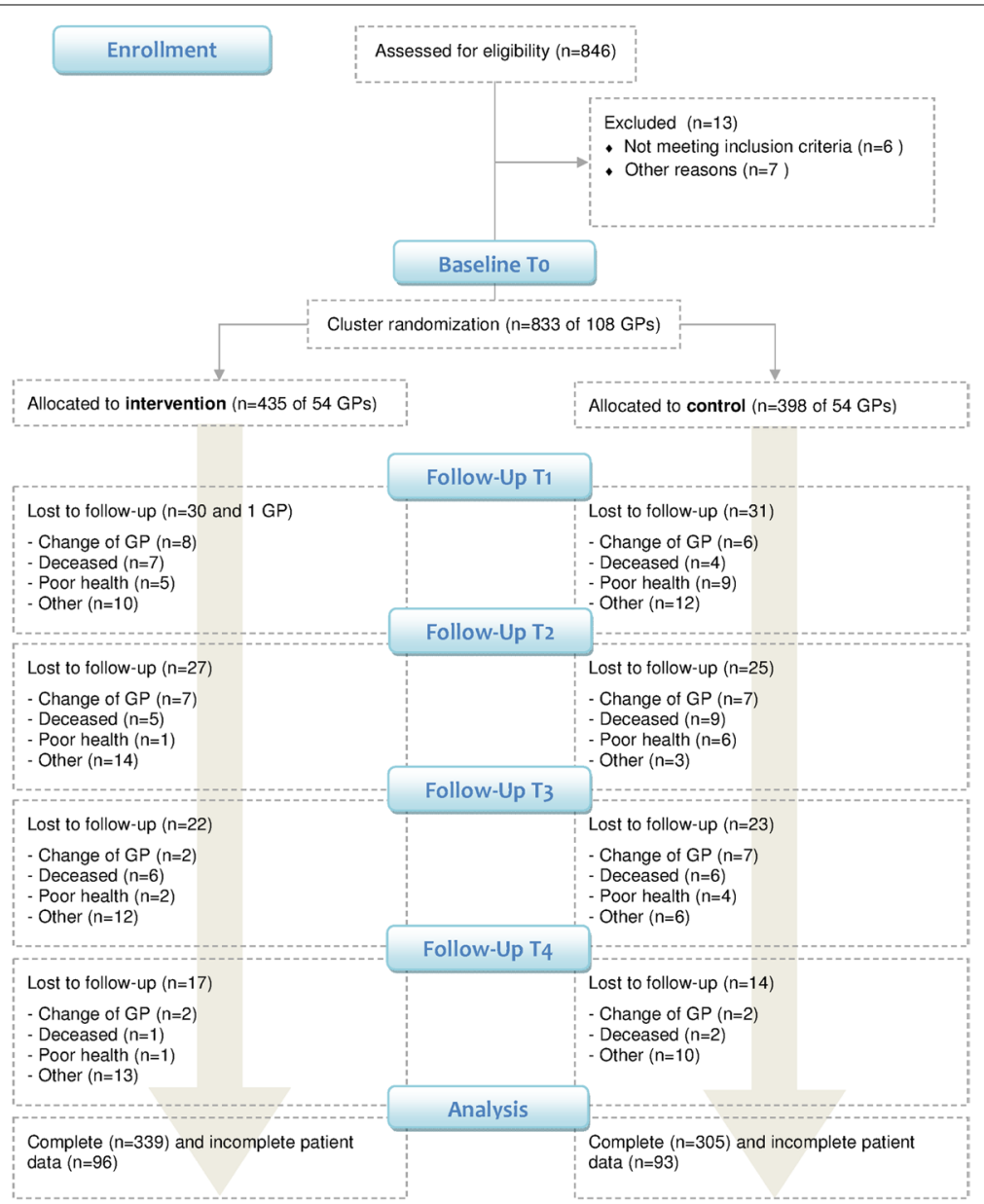

Fig. 1 Flow chart of the DEBATE trial

Table 3 Patient baseline data

\begin{tabular}{|c|c|c|c|c|c|c|}
\hline & \multicolumn{2}{|c|}{ Intervention } & \multicolumn{2}{|c|}{ Control } & \multicolumn{2}{|c|}{ Total } \\
\hline & $\mathbf{N}$ & $\%$ & $\mathbf{N}$ & $\%$ & $\mathbf{N}$ & $\%$ \\
\hline Number of patients & 435 & 52.2 & 398 & 47.8 & 833 & 100.0 \\
\hline \multicolumn{7}{|l|}{ Sex } \\
\hline Male & 241 & 55.4 & 212 & 53.3 & 453 & 54.4 \\
\hline Female & 194 & 44.6 & 186 & 46.7 & 380 & 45.6 \\
\hline Age (median) & 65.9 & & 65.8 & & 65.9 & \\
\hline \multicolumn{7}{|l|}{ Marital status ${ }^{\mathrm{a}}$} \\
\hline Single & 46 & 10.6 & 41 & 10.3 & 87 & 10.5 \\
\hline Married & 273 & 62.8 & 229 & 57.7 & 502 & 60.3 \\
\hline Divorced & 30 & 6.9 & 52 & 13.1 & 82 & 9.9 \\
\hline Widowed & 86 & 19.8 & 75 & 18.9 & 161 & 19.4 \\
\hline \multicolumn{7}{|l|}{ Living with a partner ${ }^{\mathrm{b}}$} \\
\hline Yes & 291 & 67.1 & 252 & 63.6 & 543 & 65.4 \\
\hline No & 143 & 32.9 & 144 & 36.4 & 287 & 34.6 \\
\hline Year of diagnosis (mean) & 12.4 & & 10.8 & & 11.6 & \\
\hline
\end{tabular}

a One missing value

b Three missing values 


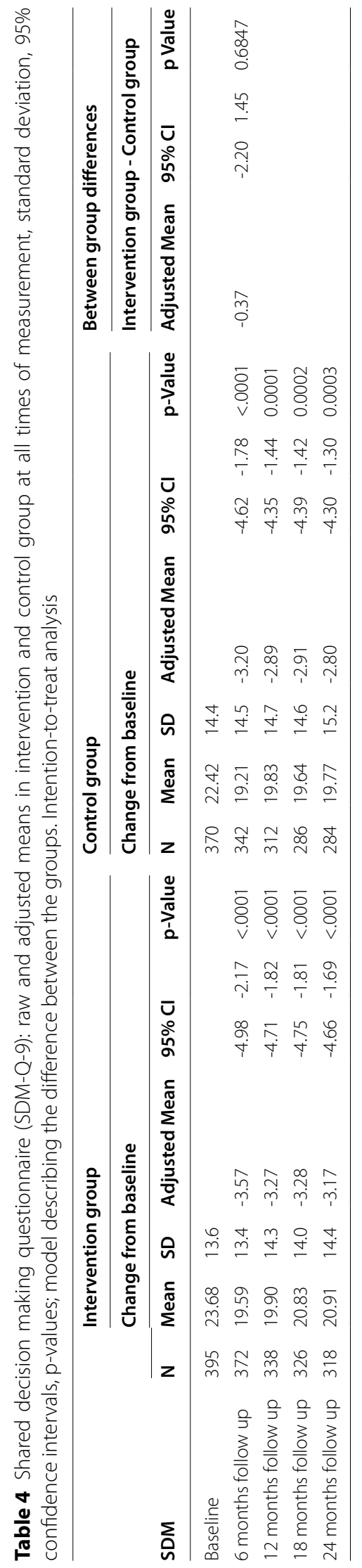




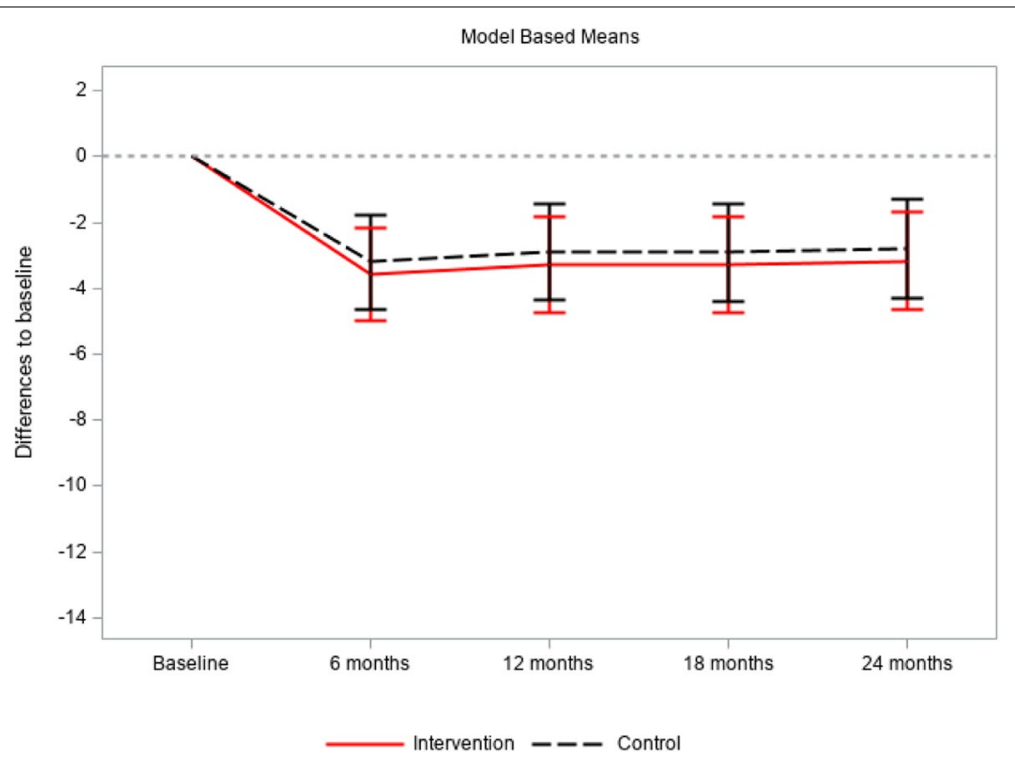

Fig. 2 Subjective shared decision making questionnaire (SDM-Q-9): adjusted means in intervention and control group at all times of measurement

increased from 1.95 at T0 to 2.11 at T4. In the control group we observed a decrease from 1.93 at T0 to 1.83 at T1 followed by an increase at T3 (2.02) and a new decline at T4 (1.97). The analyses of the single items supported this observation. However, given the exploratory character of this analysis results need to be treated cautiously.

\section{Association of patient-centeredness (PACIC-D) and subjective shared decision making (SDM-Q-9)}

Finally, a post-hoc analysis was conducted on whether change in patient-centeredness influenced extent of subjective shared decision making. The model estimated the effect of one unit increase of PACIC-D on subjective shared decision making. The model was adjusted for respective group, study centre, the SDM-Q-9 baseline value, age of initial diagnosis and time of measurement. The model showed that one unit increase of PACIC-D led to an increase of 6.52 units at the SDM-Q-9 scale (95\% CI: $5.94 ; 7.11 ; p<0.0001)$.

\section{Harms}

We did not systematically assess outcomes such as the interaction of medications, cardiovascular events, or death of patients. However, since we tested routine care against routine care with improved GP communication, we did not expect any harms.

\section{Discussion}

\section{Summary of findings}

The DEBATE trial showed that subjective patient reported shared decision making, measured by using the
SDM-Q-9 questionnaire, decreased in both intervention and control group between baseline and T4. Although the difference was statistically significant in each group, it was not between the groups. As to patient-centeredness, results were less distinct: Between baseline and T4 the score of the PACIC-D questionnaire slightly increased in both groups, however without being significantly different from baseline. Also, there was no statistically difference between intervention and control group. The analysis of the possible impact of patient-centeredness (PACIC-D) on subjective shared decision making (SDMQ-9) revealed statistically significance between the two. Thus, a one-point increase of the PACIC-D compared to baseline led to an increase of 6.52 points in the SDM-Q-9 score. Comparison of the PACIC-D results with other studies is not possible given the modification we applied to the instrument. Also, evidence on a clinically meaningful change using both, the SDM-Q-9 and the PACIC-D is limited as scores differ depending on the versions used as well as on cultural context. For these reasons, in general, comparisons are not recommended $[35,36]$.

\section{Interpretation in the context of existing literature}

The results show that the educational intervention targeting GPs in primary care setting did not lead to a relevant increase in patient reported patient-centeredness and subjective shared decision making. Over the course of the study patients rather felt less involved in shared decision making.

A recent systematic review published in 2018 included 87 studies to determine the effectiveness of interventions 


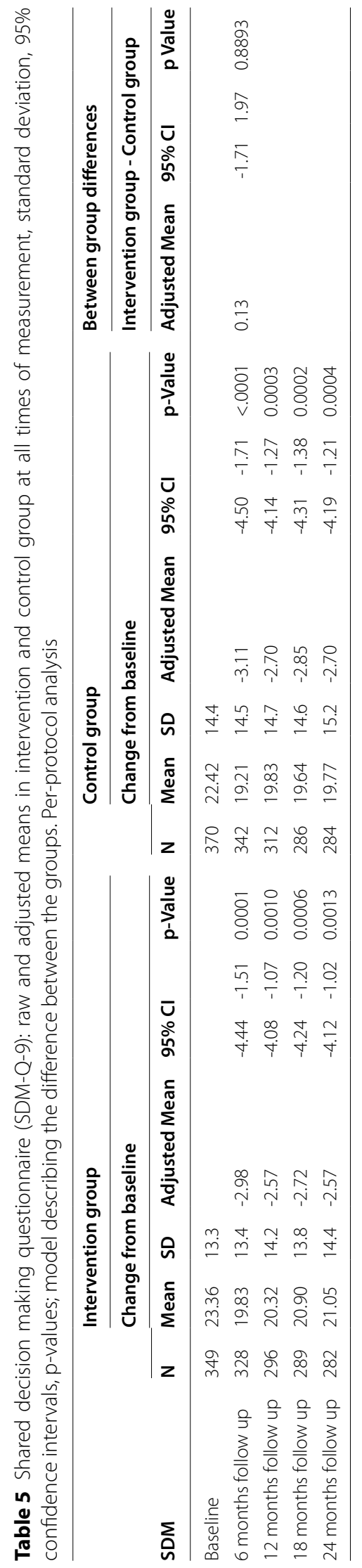




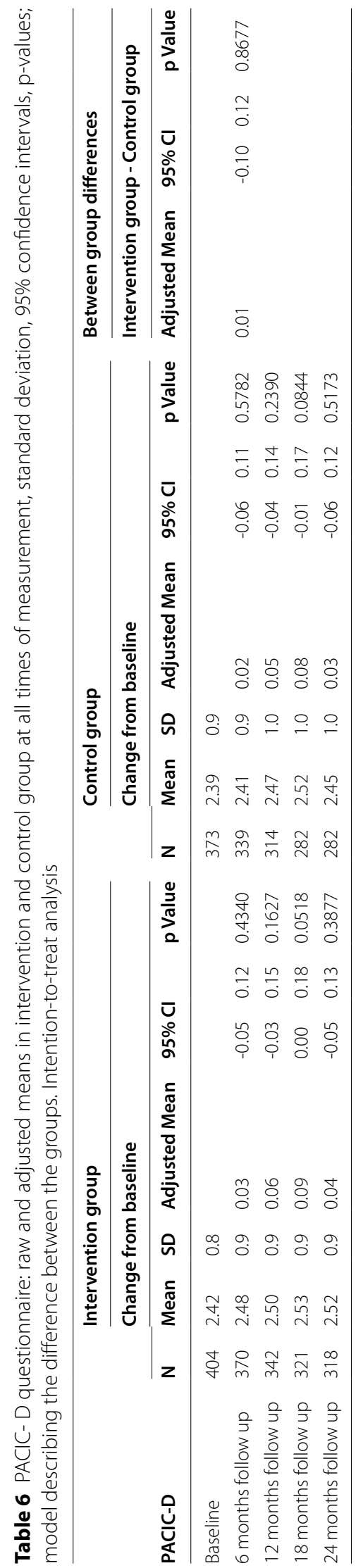




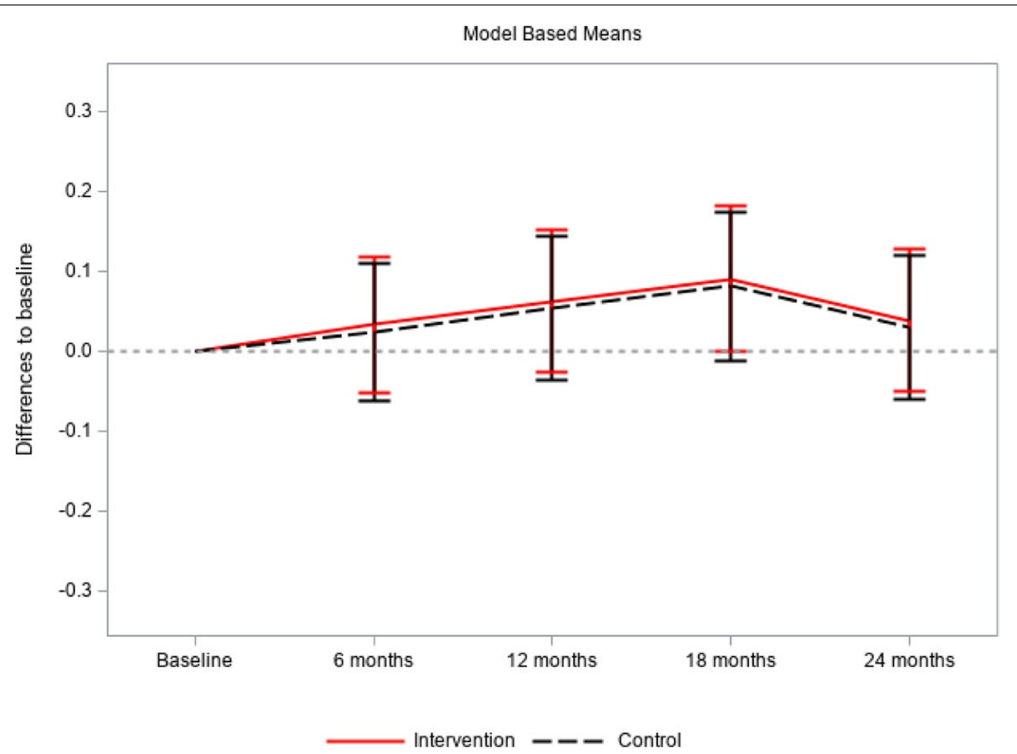

Fig. 3 PACIC-D questionnaire: adjusted means in intervention and control group at all times of measurement

on increasing the use of shared decision making by healthcare professionals (HCP). The review showed uncertain evidence. Fifteen studies included specifically evaluated the impact of interventions targeting HCPse.g., educational meetings, educational outreach visits, educational material and reminders. The certainty of evidence of these interventions was also low - regardless whether outcomes were measured by observation or reporting by patients [37]. Although there is consensus on the importance and necessity of shared decision making, is seems difficult to measure. The same seems to be true for patient centeredness.

Several studies with a similar outlay as the DEBATE trial showed concurring findings: Within a cRCT Boyd et al. measured the effect of care provided by specially trained nurses (e. g. performing assessments, monitoring care plans, coordinating care givers, and supporting family) on patients' perceived quality of health. Findings show that the mean PACIC increased over time in both groups, however the overall PACIC was higher for the intervention group. Differences between the groups were not significant [38]. In an evaluation study Szecsenyi et al. tested the effect of participation in a national disease management program for T2DM on patients' perception of chronic illness care. The study found that the difference between intervention and control group was statistically significant for the overall PACIC-score, whereby data was collected only at one time of measurement [39]. Chmiel et al. investigated the effect of a complex educational intervention targeting at practice nurses. The intervention consisted of a comprehensive educational training on medical knowledge about diabetes treatment and general communication skills. In this nested cross-sectional study, among others one outcome measure was perception of diabetes care. The authors proved significant differences between intervention and control group with a higher overall PACIC score for patients of the intervention group over the course of the study compared to those of the control group [40].

Bearing these differing findings in mind, it seems likely that the altered consciousness for patient involvement as part of the social change within the last decades requires broader instruments and concepts to measure shared decision making $[37,41,42]$.

\section{Strengths and limitations}

The DEBATE trial benefits from its long time of followup: Comparable studies often show a follow-up time considerably lower than 24 months [43, 44]. Also, patients with poorly controlled T2DM, a group that is generally hard to reach, were successfully included in the trial. Number of dropouts was stable in both groups (ca. 20\%) and reflected the original sample size calculation. Performing the intervention under real-life conditions in the setting of health care research is another strength of the study.

There are some limiting factors such as measuring subjective perception of shared decision making rather than objective (e.g., through videos of consultations) or the likely inclusion of the most receptive and motivated GPs and patients into the trial. However, in light of the effects shown above we wonder which mechanisms might have 


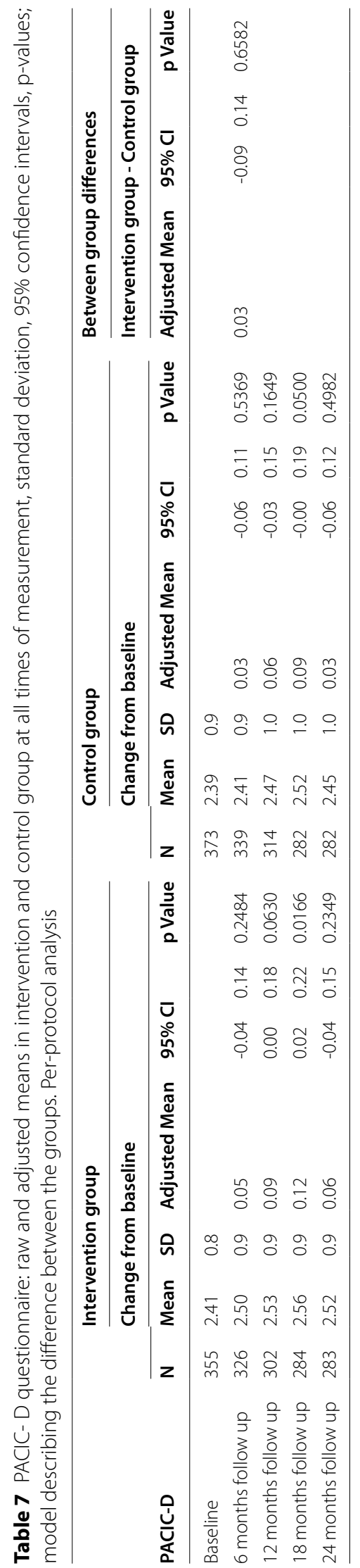


led to decreasing levels of perceived shared decision making. With inclusion into the study GPs might have been more motivated to make another attempt with their patients with poorly controlled T2DM. As a result, physicians might have treated their patients more paternalistic and might have put less emphasis on shared decision making. Improvements of HbA1c levels among both groups support this assumption [26]. Moreover, it is possible that peers visiting participating GPs were not able to initiate a change management towards shared decision making. Also, it is likely that the five measurements of shared decision making and patient-centredness over the whole study period increased patient attention towards both aspects (Hawthorne effect). This awareness might have increased patients' demand for shared decision making and patient-centredness, finally resulting in lower scores. The fact that effects were similar among the intervention and the control group support this assumption.

In retrospect, we recognize in fact discrepancies between the very intensive follow-up on the one side and the comparably surface delivery of the intervention on the other side. Although the trial might have enhanced overall awareness for poorly controlled T2DM among both, patients and physicians, it seems unlikely that it had sustainable impact on GPs counseling behavior. A different and more continuous way of intervention delivery might have been more appropriate. Unfortunately, as highlighted above, to date there is little evidence on the way shared decision making in chronic health care might be improved (and how this improvement might be measured). Légaré et al. showed that it remains uncertain if interventions targeting patients, HCPs or both increase shared decision making [37]. In their systematic review of shared decision making and patient outcomes Shay and Elston Lafata conclude that it remains unclear what leads a patient to report a decision as shared and thus how to foster shared decision making [45].

\section{Clinical impact}

Results did not show a significant difference in subjective extent of shared decision making and patient-centeredness between intervention and control group. In this respect the intervention scheme did not have an effect. Nonetheless, intensive and individual follow-ups, which exceeded the care offered through Disease Management Programmes by far, seem to be supportive. Besides the documentation of laboratory values and the definition of therapeutic outcomes, in particular the patients' individual perception of and problems with their disease were of interest. The findings might motivate general practitioners to continuously engage with this challenging group of patients and to remain open-minded for new communication approaches, which can help to achieve a continuation of therapy.

\section{Future research}

Instead of delivering physician-focused interventions, future studies might investigate the effect of interventions addressing and empowering both patients and physicians to foster shared decision making and patientcenteredness in diabetes care. In doing so, studies should increasingly focus on the black box "intervention" in regard to their effectiveness or lack thereof. For example, qualitative measures could determine possible hurdles of GPs and patients in changing behaviour.

\section{Conclusions}

The DEBATE study did not result in improved care for patients with poorly controlled T2DM of the trialled intervention group in comparison to the control group. Nonetheless, an improvement in care for this patient population in the primary care setting is desirable. For this reason alone, GPs should be motivated to further engage in this group of patients.

\section{Abbreviations \\ GP: General Practitioner; HCP: Health care professional; ITT: Intention-to-treat; PP: Per Protocol; T2DM: Type 2 Diabetes Mellitus. \\ Acknowledgements \\ We thank all patients and general practitioners for their participation in the trial. And we are deeply grateful to all physicians supporting the intervention by peer-visiting GPS.}

\section{Authors' contributions}

AA and SW designed and managed the project. AW, SS, ED, SL, MP, CH, GF were responsible for setting up the trial, recruiting participants, implementing the intervention and collecting data. $\mathrm{AD}$ and $\mathrm{KW}$ performed data analyses. AW, CL and SS wrote the manuscript. All authors read, critically revised and approved the final manuscript.

\section{Funding}

Open Access funding enabled and organized by Projekt DEAL. The trial was funded by the German Ministry of Education and Research (Reference: 01GX1041A). The funding body had no influence on the design of the study; collection, analysis, and interpretation of data; and in writing the manuscript.

\section{Availability of data and materials}

The datasets generated and analyzed during the current study are available from the corresponding author on reasonable request.

\section{Declarations}

Ethic approval and consent to participate

The Ethics Committee at Rostock University Medical Centre approved the trial on May 25 ${ }^{\text {th }}, 2011$ under the reference A 2011-59. Ethics Committees at the Medical Faculty of the Heinrich-Heine-University Dusseldorf (July $19^{\text {th }}, 2011$, reference 3676 ) and at the University of Witten/Herdecke (July $12^{\text {th }}, 2011$, reference $78 / 2011$ ) confirmed scientific adequacy. Consent obtained from participants was written.

Consent for publication

Not applicable. 


\section{Competing interests}

The authors declare no conflict of interest.

\section{Author details}

${ }^{1}$ Institute of General Practice, Rostock University Medical Centre, Doberaner Str. 142, 18057 Rostock, Germany. ${ }^{2}$ Institute of Medical Biometry and Epidemiology, Medical Centre Hamburg-Eppendorf, Martinistraße 52, 20246 Hamburg, Germany. ${ }^{3}$ Institute of General Practice (Ifam), Medical Faculty, Centre for Health \& Society (Chs), Heinrich-Heine University Düsseldorf, Moorenstr. 5, 40225 Düsseldorf, Germany.

Received: 6 May 2020 Accepted: 12 April 2021

Published online: 15 May 2021

\section{References}

1. Shaw J, Sicree R, Zimmet P. Global estimates of the prevalence of diabetes for 2010 and 2030. Diabetes Res Clin Pract. 2010;87(1):4-14.

2. Robert-Koch-Institut. Diabetes mellitus - DEGS1-Faktenblatt. Studie zur Gesundheit Erwachsener in Deutschland (DEGS1). 2016.

3. World Health Organization. Global report on diabetes. 2016. World Health Organization.

4. Chen L, Magliano DJ, Zimmet PZ. The worldwide epidemiology of type 2 diabetes mellitus-present and future perspectives. Nat Rev Endocrinol. 2011:8(4):228-36

5. Danaei G, Finucane MM, Lu Y, Singh GM, Cowan MJ, Paciorek CJ, Lin JK, Farzadfar F, Khang YH, Stevens GA, Rao M, Ali MK, Riley LM, Robinson CA, Ezzati M. National, regional, and global trends in fasting plasma glucose and diabetes prevalence since 1980: systematic analysis of health examination surveys and epidemiological studies with 370 countryyears and 2.7 million participants. Lancet. 2011;378(9785):31-40.

6. Khan MAB, Hashim MJ, King JK, Govender RD, Mustafa H, Kaabi JA. Epidemiology of type 2 diabetes - global burden of disease and forecasted trends. J Epidemiol Glob Health. 2020;10(1):107-11.

7. Zentralinstitut für die kassenärztliche Versorgung (Zi). DMP-Qualitätsbericht Nordrhein 2013. 2013. Düsseldorf.

8. Frei A, Herzog S, Woitzek K, Held U, Senn O, Rosemann T, Chmiel C. Characteristics of poorly controlled type 2 diabetes patients in Swiss primary care. Cardiovasc Diabetol. 2012:11:70.

9. Wilm S, Abholz HH, Gummersbach E, Icks A, Pentzek M. Menschen mitschlechteingestelltem typ-2-diabetes - GesundheitspsychologischeCharakterisierung. Diabetologe. 2014;10:2000-6.

10. Delamater AM, Jacobson AM, Anderson BJ, Cox D, Fisher L, Lustman $P$, Rubin R, Wysocki T. Psychosocial therapies in diabetes: report of the Psychosocial Therapies Working Group. Diabetes Care. 2001:24(7):1286-92.

11. Peyrot M, McMurry JF, Kruger DF. A biopsychosocial model of glycemic control in diabetes: stress, coping and regimen adherence. J Health Soc Behav. 1999;40(2):141-58

12. Deakin T, McShane CE, Cade JE, Williams RDRR. Group based training for self-management strategies in people with type 2 diabetes mellitus. Cochrane Database Syst Rev. 2005;(2):CD003417. https://doi.org/10.1002/ 14651858.CD003417.pub2.

13. Rothenbacher D, Brenner H, Rüter G. Typ-2-diabetes-mellitus: Betreuung von chronischKranken in der Hausarztpraxis. Dtsch Arztebl. 2005;102(36):2408-12.

14. Rushforth B, McCrorie C, Glidewell L, Midgley E, Foy R. Barriers to effective management of type 2 diabetes in primary care: qualitative systematic review. Br J Gen Pract. 2016;66(643):e114-27.

15. Abholz HH, Egidi G, Rüter G. Der Umgangmit „schlechteingestellten” patientenmit typ-2-diabetes - EineBeobachtungsstudie von Hausärzten. Z Allg Med. 2016;92(3):109-15.

16. Wollny A, Pentzek M, Herber OR, Abholz H-H, In der Schmitten J, Icks A, Wilm S, Gummersbach E. General practitioners' attitudes towards patients with poorly controlled type 2 diabetes: a qualitative study. BMC Fam Pract. 2018;19:49.

17. Crosson J, Heisler M, Subramanian U, Swain B, Davis GJ, Lasser N, Ross S, Schmittdiel JA, Onyemere K, Tseng C-W. Physicians' perceptions of barriers to cardiovascular disease risk factor control among patients with diabetes: results from the translating research into action for diabetes (TRIAD) study. J Am Board Fam Med. 2010;23(2):171-8.
18. Wens J, Vermeire E, Van Royen P, Sabbe B, Denekens J. GPs' perspectives of type 2 diabetes patients' adherence to treatment: a qualitative analysis of barriers and solutions. BMC Fam Pract. 2005:6(1):20.

19. Noor Abdulhadi NM, Al-Shafaee MA, Wahlström R, Hjelm K. Docotors' and nurses' view on patient care for type 2 diabetes: an interview study in primary health care in Oman. Prim Health Care Res Dev. 2013;14(3):258-69.

20. Agarwal G, Nair K, Cosby J, Dolovich L, Levine M, Kaczorowski J, Butler C, Burns S. GPs'approach to insulin prescribing in older patients: a qualitative study. Br J Gen Pract. 2008;58(553):569-75.

21. Elwyn G, Edwards A, Mowle S, Wensing M, Wilkinson C, Kinnersley P, Grol R. Measuring the involvement of patients in shared decision-making: a systematic review of instruments. Patient Educ Couns. 2001;43(1):5-22.

22. Charles C, Whelan T, Gafni A. What do we mean by partnership in making decisions about treatment? BMJ. 1999:319(7212):780-2.

23. Herber O, Wollny A, Pentzek M, Abholz HH, Icks A, Wilm S. What do family physicians tell about their patients with diabetes mellitus type 2 ? Possible reasons for insufficient blood glucose control. Z Allg Med. 2010;86(5):203-8.

24. Zoffmann V, Harder I, Kirkevold M. A person-centered communication and reflection model: sharing decision-making in chronic care. Qual Health Res. 2008;18(5):670-85.

25. Altiner A, Donner-Banzhoff N. Chronisch krank sein - subjektive Aspekte. Z Allg Med. 2008;84(4):157-60.

26. Wollny A, Altiner A, Daubmann A, Drewelow E, Helbig C, Löscher S, Pentzek M, Santos S, Wegscheider K, Wilm S, Löffler C. Patient-centered communication and shared decision making to reduce $\mathrm{HbA} 1 \mathrm{c}$ levels of patients with poorly controlled type 2 diabetes mellitus - results of the cluster-randomized controlled DEBATE trial. BMC Fam Pract. 2019;20(1):87.

27. Drewelow E, Wollny A, Pentzek M, et al. Improvement of primary health care of patients with poorly regulated diabetes mellitus type 2 using shared decision-making - the DEBATE trial. BMC Fam Pract. 2012;13:88.

28. Sadowski EMEC, Keller H, Krones T, Sönnichsen AC, Baum E, DonnerBanzhoff N. Evaluation komplexer Interventionen: Implementierung von ARRIBA-Herz, einer Beratungsstrategie für die Herz-Kreislaufprävention [Evaluation of complex interventions: implementation of ARRIBAHerz, a counseling strategy for coronary prevention]. Z Allg Med. 2005:81:429-34.

29. Arriba. [Available from: http://arriba-hausarzt.de/].

30. Kriston LSI, Hölzel L, Simon D, Loh A, Härter M. The 9-item shared decision making questionnaire (SDM-Q-9) developed and psychometric properties in a primary care sample. Patient Educ Couns. 2010;80:94-9.

31. Gugiu PC, Coryn C, Clark R, et al. Development and evaluation of the short version of the patient assessment of chronic illness care instrument. Chronic IIIn. 2009;5(4):268-76.

32. Glasgow RE, Wagner EH, Schaefer J, Mahoney LD, Reid RJ, Greene SM. Development and validation of the patient assessment of chronic illness care (PACIC). Med Care. 2005;43(5):436-44

33. Rosemann T, Laux G, Droesemeyer S, Gensichen J, Szecsenyi J. Evaluation of a culturally adapted German version of the patient assessment of chronic illness care (PACIC 5A) questionnaire in a sample of osteoarthritis patients. J Eval Clin Pract. 2007;13(5):806-13.

34. Goetz K, Freund T, Gensichen J, Miksch A, Szecsenyi J, Steinhaeuser J. Adaptation and psychometric properties of the PACIC short form. Am J Manag Care. 2012;18(2):e55-60.

35. Arditi C, Iglesias K, Peytremann-Bridevaux I. The use of the patient assessment of chronic illness care (PACIC) instrument in diabetes care: a systematic review and meta-analysis. Int J Qual Health Care. 2018;30(10):743-50

36. Doherr H, Christalle E, Kriston L, Härter M, Scholl I. Use of the 9-item shared decision making questionnaire (SDM-Q-9 and SDMQ-Doc) in intervention studies - a systematic review. PLoS ONE. 2017;12(3):e0173904

37. Légaré F, Adekpedjou R, Stacey D, Turcotte S, Kryworuchko J, Graham ID, Lyddiatt A, Politi MC, Thomson R, Elwyn G, Donner-Banzhoff N. Interventions for increasing the use of shared decision making by healthcare professionals. Cochrane Database Syst Rev. 2018;19(7):CD006732.

38. Boyd CM, Reider L, Frey K, Scharfstein D, Leff B, Wolff J, Groves C, Karm L, Wegener S, Marsteller J, Boult C. The effects of guided care on the perceived quality of health care for multi-morbid older persons: 18-month 
outcomes from a cluster-randomized controlled trial. J Gen Intern Med. 2010;25(3):235-42.

39. Szecsenyi J, Rosemann T, Joos S, Peters-Klimm F, Miksch A. German diabetes disease management programs are appropriate for restructuring care according to the chronic care model: an evaluation with the patient assessment of chronic illness care instrument. Diabetes Care. 2008;31(6):1150-4.

40. Chmiel C, Giewer I, Frei A, Rosemann T. Four-year long-term follow-up of diabetes patients after implementation of the chronic care model in primary care: a cross-sectional study. Swiss Med Wkly. 2017;147:w14522.

41. Lehman R. Shared decision making - essential but hard to measure. The BMJ opinion, 2018. Available at: https://blogs.bmj.com/bmj/2018/ 08/10/richard-lehman-shared-decisionmaking-essential-hard-measure/ (Accessed 19 Feb 2019).

42. Elwyn G, Frosch DL, Kobrin S. Implementing shared decision making: consider all the consequences. Implement Sci. 2016;88(11):114
43. Murphy ME, Byrne M, Galvin R, Boland F, Fahey T, Smith S. Improving risk factor management for patients with poorly controlled type 2 diabetes: a systematic review of healthcare interventions in primary care and community settings. BMJ Open. 2017;7(8):e015135.

44. Seidu S, Walker NS, Bodicoat DH, Davies MJ, Khunti K. A systematic review of interventions targeting primary care or community based professionals on cardio-metabolic risk factor control in people with diabetes. Diabetes Res Clin Pract. 2016;113:1-13.

45. Shay LA, Elston LJ. Where is the evidence? A systematic review of shared decision making and patient outcomes. Med Decis Making. 2015;35(1):114-31.

\section{Publisher's Note}

Springer Nature remains neutral with regard to jurisdictional claims in published maps and institutional affiliations.
Ready to submit your research? Choose BMC and benefit from:

- fast, convenient online submission

- thorough peer review by experienced researchers in your field

- rapid publication on acceptance

- support for research data, including large and complex data types

- gold Open Access which fosters wider collaboration and increased citations

- maximum visibility for your research: over $100 \mathrm{M}$ website views per year

At BMC, research is always in progress.

Learn more biomedcentral.com/submissions 